\title{
iTRAQ-based quantitative analysis of protein mixtures with large fold change and dynamic range
}

\author{
Juan Casado-Vela ${ }^{1}$ María José Martínez-Esteso², Eva Rodriguez ${ }^{1}$, Eva Borrás ${ }^{3}$,Felix Elortzal, \\ Roque Bru-Martínez ${ }^{2}$
}

${ }^{1}$ Proteomics Platform. CIC bioGUNE, CIBERehd, ProteoRed. Technology Park of Bizkaia, Building 800. Derio, Spain. ${ }^{2}$ Grupo de Proteómica y Genómica Funcional de Plantas. Departamento de Agroquímica y Bioquímica. Facultad de Ciencias. Universidad de Alicante. Spain. ${ }^{3}$ Servicio Proteómica. Universidad Pompeu Fabra. Parc de Recerca Biomèdica de Barcelona. Dr Aiguader 88. 08003 Barcelona, Spain

Quantitation of changes in protein abundance is key to discovering novel biomarkers. Currently, reverse phase liquid chromatography coupled to tandem mass spectrometry (HPLC-MS/MS) can be used to quantify changes in protein expression levels. Nevertheless, quantitative analysis of protein mixtures by HPLC-MS/MS is still hampered by the wide range of protein expression levels, the high dynamic range of protein concentrations and the lack of reliable quantitation algorithms. In this context, we describe two different samples (4-protmix and 8-protmix) suitable for relative protein quantitation using isobaric Tags for Relative and Absolute Quantitation (iTRAQ). Using the 4-protmix, relative protein changes of up to 24-fold were measured. The 8-protmix allowed the quantitation of the relative protein changes in a mixture of proteins within the range of two orders of magnitude in concentration and 10-fold differences in relative abundance.

The two reference samples proposed here (4-protmix and 8-protmix) cover a wide range of protein fold changes and protein concentrations, respectively, which can be used to optimize the settings used during acquisition with different mass spectrometers and to test the performance of different quantitation algorithms used for iTRAQ experiments.

Three technical replicates corresponding to 800 fmol of 4-protmix and 8-protmix were analyzed by HPLC-MS/MS using two platforms, a ChipLC coupled to a 6530 Q-ToF (Agilent Technologies) and a 1200 nano-HPLC (Agilent Technologies) coupled to an LTQ Orbitrap (Thermo Fisher Scientific). As a result, the analysis of the 4-protmix and the 8-protmix led to the successful identification and quantitation of all proteins in the samples (Tables 1 and 2).

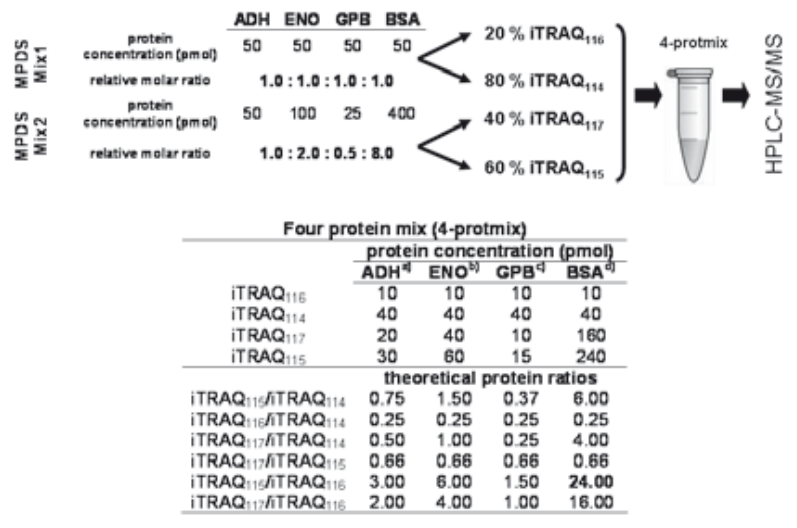

Figure 1. Schematic view of the preparation of the 4-protmix.

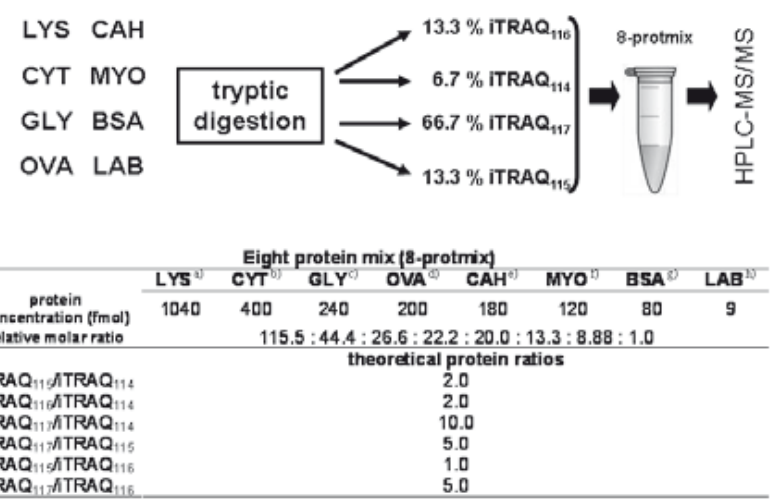

Figure 2. Schematic view of the preparation of the 8-protmix.

As shown here, we were able to detect up to 24 -fold changes in protein ratios. The standard deviation $(\sigma)$ was typically lower than 1.0 but ranged from $\sigma=0.01$ to $\sigma=6.37$. The highest $\sigma$ corresponded to the highest protein fold change in our samples (24-fold). 
Table 1. 4-protmix iTRAQ protein relative quantitation. A: ChipLC coupled to a 6530 Q-ToF and analysed using MASCOT. B: 1200 nanoHPLC coupled to LTQ Orbitrap and analysed using Proteome Discover.

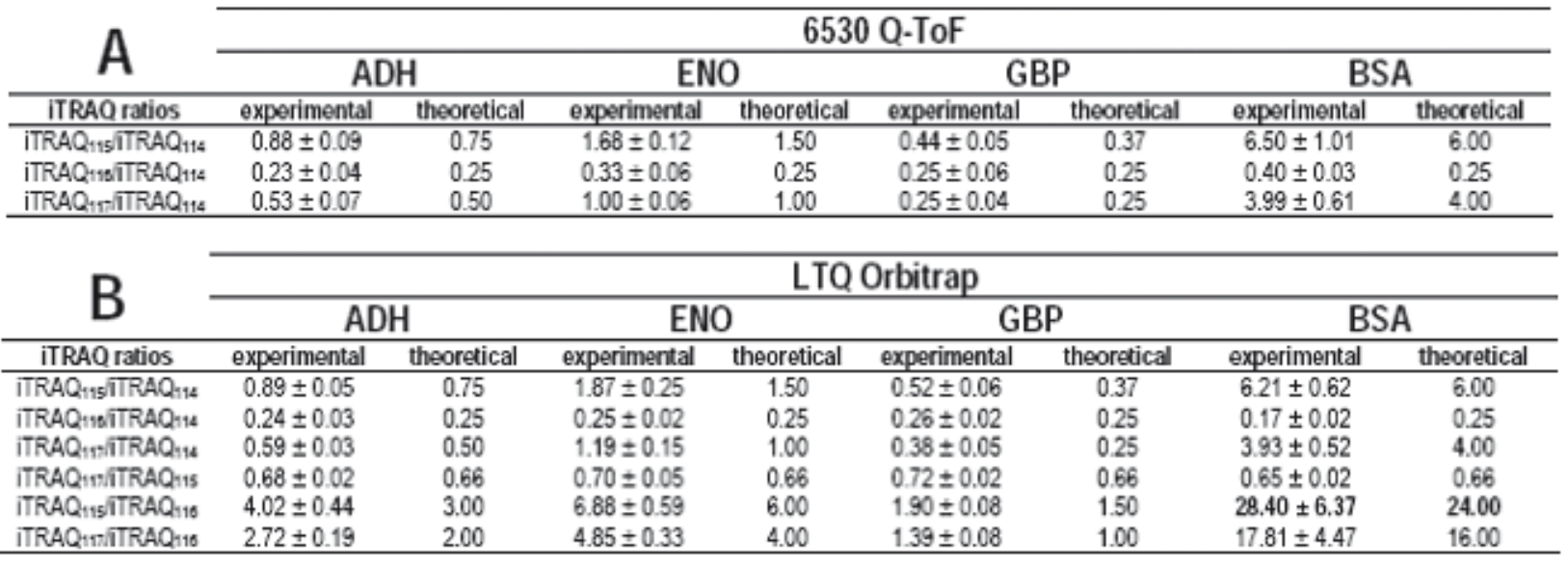

Table 2. 8-protmix iTRAQ protein relative quantitation measured with LTQ Orbitrap and analysed using Proteome Discover

\begin{tabular}{|c|c|c|c|c|c|c|c|c|c|}
\hline \multicolumn{10}{|c|}{ Eight protein mix (8-protmix) } \\
\hline & [YS ] & $\mathrm{CYT}^{\left.{ }^{0}\right]}$ & GLYc) & OVAd & $\mathrm{CAH}^{*}$ & MYO? & BSA 9 & $\mathrm{LAB}^{\mathrm{n})}$ & $\begin{array}{c}\text { theoretical protein } \\
\text { ratios }\end{array}$ \\
\hline$I_{T R A Q_{11 S} / T R A Q_{114}}$ & $2.15 \pm 0.08$ & $2.18 \pm 0.09$ & $2.48 \pm 0.19$ & $2.54 \pm 0.11$ & $2.20 \pm 0.08$ & $2.36 \pm 0.14$ & $2.19 \pm 0.05$ & $1.85 \pm$ n.c. & 2.0 \\
\hline ITRAQ $11 d / T R A Q_{114}$ & $2.12 \pm 0.11$ & $2.16 \pm 0.10$ & $220 \pm 0.11$ & $2.21 \pm 0.15$ & $2.19 \pm 0.15$ & $2.15 \pm 0.43$ & $2.19 \pm 0.06$ & $1.93 \pm$ n.c. & 2.0 \\
\hline ITRAQ $111 / T R A Q_{114}$ & $11.54 \pm 0.54$ & $11.98 \pm 0.74$ & $12.40 \pm 1.49$ & $13.97 \pm 1.27$ & $12.33 \pm 0.24$ & $12.61 \pm 0.92$ & $12.14 \pm 0.28$ & $8.79 \pm n . c$. & 10.0 \\
\hline ITRAQ $117 / T R A Q_{115}$ & $5.65 \pm n . c$. & $5.20 \pm 0.21$ & $5.25 \pm 0.06$ & $5.48 \pm 0.40$ & $6.12 \pm 0.36$ & $5.52 \pm 0.25$ & $5.69 \pm 0.31$ & n.e. & 5.0 \\
\hline ITRAQ $115 / T R A Q_{115}$ & $1.04 \pm 0.04$ & $1.00 \pm 0.01$ & $1.10 \pm 0.09$ & $1.16 \pm 0.07$ & $1.00 \pm 0.02$ & $1.13 \pm 0.16$ & $1.02 \pm 0.04$ & $0.96 \pm$ n.c. ${ }^{2}$ & 1.0 \\
\hline $\mathrm{ITRA}_{111} \sqrt{\mathrm{TR}} \mathrm{RQ}_{116}$ & $5.45 \pm 0.40$ & $5.43 \pm 0.21$ & $5.51 \pm 0.28$ & $6.48 \pm 1.41$ & $5.74 \pm 0.17$ & $5.96 \pm 0.65$ & $5.64 \pm 0.02$ & $4.56 \pm n . c^{9}$ & 5.0 \\
\hline
\end{tabular}

In conclusion, we described two different samples suitable for iTRAQ analysis that can be used to assess the performance of different mass spectrometers and quantitation algorithms. A considerable part of the proteome of a typical biological sample falls within the fold change and dynamic range windows of these two samples.

\section{Evaluation of MSE based protein quantification methods}

Kerman Aloria ${ }^{1}$, Miren Josu Omaetxebarria ${ }^{2}$, Mikel Azkargorta ${ }^{2 *}$, Johannes P. C. Vissers ${ }^{4}$, Asier Fullaondo ${ }^{5}$, Jesus M. Arizmendi ${ }^{1,2}$

${ }^{1}$ Proteomics Core Facility-SGIker (ProteoRed). University of the Basque Country. Leioa, Spain. ${ }^{2}$ Department of Biochemistry and Molecular Biology. University of the Basque Country. Leioa, Spain. ${ }^{3}$ Proteomics Core Facility. CIC bioGUNE. Derio, Spain. ${ }^{4}$ Waters Corporation. Manchester, United Kingdom. ${ }^{5}$ Department of Genetics, Physical Anthropology and Animal Physiology. University of the Basque Country. Leioa, Spain

* Current address: Proteomics Core Facility. CIC bioGUNE. Derio, Spain

LC-MS based protein quantification methods have recently gained popularity as powerful technologies capable of addressing protein expression analysis in complex samples. As an alternative to isotope labeling methodologies, which are samplenumber and sample-type dependant and require special labeling chemistries, label-free approaches afford greater experimental design and less sample 